\title{
Alimentação de transição infantil: conhecer para educar
}

\author{
Infant transition feeding: learn to educate
}

\author{
Pábula Parente Correia ${ }^{1}$, Sandra Mara Pimentel Duavy Pereira², \\ Lorena Andréa Maciel Honor de Brito ${ }^{3}$
}

\begin{abstract}
RESUMO
Objetivo: Identificar os conhecimentos dos agentes comunitários de saúde de uma Equipe de Estratégia de Saúde da Família acerca de informações sobre alimentação de transição infantil, também denominada alimentação complementar, no período da lactância. Tal identificação poderá subsidiar a elaboração de uma estratégia educativa para aperfeiçoamento desses conhecimentos.

Materiais e Métodos: Estudo qualitativo, exploratório, com delineamento do tipo pesquisa-ação, construído a partir das entrevistas semiestruturadas realizadas com os agentes comunitários de saúde, cujos dados foram analisados pela técnica de análise de conteúdo de Bardin. Posteriormente, utilizou-se uma estratégia educativa com os participantes, a qual consistiu em uma palestra sobre a temática do estudo. Finalmente, aplicou-se um questionário com estes para avaliar o impacto da estratégia implementada.

Resultados: Identificou-se conhecimento deficiente sobre os fatores imprescindíveis à oferta de uma alimentação complementar adequada, bem como sobre os fatores condicionantes dos problemas nutricionais mais comuns. Contudo, a aplicação do questionário, após a capacitação, evidenciou melhora no nível de conhecimento por parte dos sujeitos quanto à temática alimentação de transição.

Conclusão: É deficiente o conhecimento dos agentes comunitários de saúde em relação à alimentação de transição infantil, apontando para a necessidade de aperfeiçoamento frente à temática por parte desses profissionais de saúde, capaz de propiciar melhora na qualidade das informações e, por conseguinte, das orientações prestadas à comunidade. Além disso, o desfecho favorável alcançado com a realização da estratégia educativa proposta pelo estudo fortalece a importância de haver essa educação continuada.
\end{abstract}

Palavras-chave: lactente; alimentação complementar; agente comunitário de saúde.

\section{ABSTRACT}

Objective: To identify the knowledge of the community health agents from one Family Strategy Health Team about the information on infant transition feeding, also called supplementary food, in the lactation period. Such identification may support the development of an educational strategy to improve these skills.

Materials and Methods: Qualitative, exploratory study, with an action-research type design, conducted using semistructured interviews realized with community health agents. Data were analyzed using the Bardin content analysis technique. Afterwards, we used an educational strategy with the participants, which consisted in a lecture on the topic of the study. Finally, a questionnaire was applied to the participants to evaluate the impact of the strategy implemented.

Results: We have identified poor knowledge about the essential factors needed for an appropriate complementary feeding, as well as upon the most common conditional factors of nutritional problems. However, the questionnaire application, after the educational strategy, showed an improvement in the knowledge level of the subjects on the issue of feeding transition.

Conclusion: The knowledge of the community health agents in relation to infant transitional feeding is poor, pointing to the need of improvement in the theme by the health professionals, which could provide a better quality of the information, and, consequently, of the orientation provided to the community. Furthermore, the favorable outcome achieved due to the educational strategy proposed in the study strengthens the importance of having this continuing education.

Keywords: infant; supplementary feeding; community health workers.

\footnotetext{
${ }^{1}$ Enfermeira. Especialista em Estratégia de Saúde da Família pela Universidade Estadual Vale do Acaraú. Residente em Enfermagem na área de Cardiologia pela Universidade Estadual do Pernambuco, Recife/PE.

${ }^{2}$ Nutricionista. Mestre em Bioquímica pela Universidade Federal do Rio Grande do norte. Professora da Universidade Regional do Cariri, Crato/CE.

${ }^{3}$ Nutricionista. Especialista em Saúde Coletiva pela Universidade Estadual do Ceará. Coordenadora do Sistema de Vigilância Alimentar e Nutricional (SISVAN) do município de Juazeiro do Norte/CE.
} 


\section{INTRODUÇÃO}

Durante o primeiro ano de vida as intensas alterações físicas, psicomotoras e cognitivas vivenciadas pela criança trazem crescentes e diferenciadas necessidades nutricionais a serem supridas pela mãe ou cuidador, e que vão desde o período de aleitamento até a introdução de novos alimentos (transição alimentar), podendo gerar nesses, dúvidas e ansiedade quanto ao correto fornecimento de uma alimentação ótima ${ }^{1}$.

A oferta adequada de alimentação complementar ou alimentação de transição, definida como introdução de outros alimentos sólidos e líquidos em adição ao leite materno, a partir dos seis meses de idade, é imprescindível e precisa dar-se paulatinamente, uma vez que seu início precoce ou tardio pode acarretar carências ou excessos alimentares, contribuindo como fator de risco para deficiência de nutrientes, desnutrição ou sobrepeso, podendo predispor o indivíduo a doenças crônicas na idade adulta ${ }^{2}$.

Portanto, é de suma importância conseguir-se uma correta abordagem familiar por parte dos profissionais de saúde, especialmente aqueles que atuam na atenção básica e que estão inseridos na Estratégia de Saúde da Família (ESF), com vistas a promover, em cooperação com a família, um período de transição alimentar sem traumas ou intercorrências, garantindo segurança nutricional na idade mais tenra, na qual a maturação das estruturas e funções orgânicas do bebê requer energia e disponibilidade de nutrientes diversos ${ }^{3}$.

Dentre os profissionais que atuam na equipe de ESF, - Agente Comunitário de Saúde (ACS) tem como principal competência, segundo preconizações do Ministério da Saúde, realizar atividades de prevenção de doenças e promoção da saúde por meio do cumprimento de atribuições básicas como, o estabelecimento do contato permanente com as famílias, desenvolvendo ações educativas planejadas pela equipe e a escuta qualificada das necessidades dos usuários em todas as ações, proporcionando atendimento e estabelecimento do vínculo entre equipe de saúde e população ${ }^{4}$.

Portanto, enquanto importante elo entre os serviços de saúde e a comunidade, o ACS poderá contribuir através do repasse de informações adequadas, para a construção de hábitos alimentares saudáveis. Assim, pode-se dizer que trabalhar previamente com o ACS sobre alguma ferramenta de melhoramento das condições da comunidade, poderá contribuir para a produção de maiores impactos na intervenção ${ }^{5}$.

Neste sentido, reconhecendo a relevância de inserir os ACSs na realização de uma educação em saúde eficaz, capaz de imprimir mudanças em longo prazo, e entendendo os desafios do período de transição alimentar, pretende-se com o presente estudo identificar os conhecimentos dos ACSs acerca de informações sobre alimentação de transição infantil. Uma vez conhecidas as principais dificuldades ou dúvidas referentes ao tema, por parte dos ACSs, propõe-se uma capacitação com estes, no intuito de motivá-los à busca de novas estratégias que produzam aprendizado na comunidade, garantindo a continuidade e qualidade das informações passadas.

\section{MATERIAIS E MÉTODOS}

Tratou-se de um estudo exploratório, com delineamento do tipo pesquisa-ação, utilizando-se abordagem qualitativa, realizado em uma Unidade Básica de Saúde do município de Juazeiro do Norte-CE. Esta unidade é responsável pelo acompanhamento de 56 lactentes, o que caracteriza uma grande demanda se comparada ao perfil das outras unidades básicas da mesma cidade.

Os sujeitos da pesquisa foram os oito ACSs desta equipe da Estratégia de Saúde da Família, os quais atendiam aos seguintes critérios de inclusão: ser profissional agente comunitário de saúde que estivesse trabalhando há, no mínimo, seis meses na referida unidade, e aceitar participar voluntariamente do estudo.

Os dados foram coletados por meio de entrevista semiestruturada, na qual se indagava sobre os aspectos de uma alimentação complementar adequada, bem como o que caracterizaria uma alimentação complementar inapropriada e quais seriam suas consequências. Após transcrição das entrevistas, as informações foram ordenadas conforme leitura do material, sendo analisadas pela Técnica de Análise de Conteúdo, segundo Bardin ${ }^{6}$, seguindo-se as etapas de pré-análise; exploração do material; e o tratamento dos 
resultados obtidos, a inferência e a interpretação.

Assim, inicialmente os dados foram organizados e sistematizados após leitura flutuante das informações coletadas. Em seguida, sucedeu-se à exploração do material por meio de leitura exaustiva e codificação dos dados, transformado-os em unidades de compreensão do texto, considerando-se como unidade base o tema. Essas unidades, denominadas unidades de análise temática foram submetidas às regras de enumeração, categorização e eventual subcategorização. Por fim, foram feitas reflexões e interpretações acerca das categorias e subcategorias.

Portanto, em função das semelhanças entre seus conteúdos, emergiram categorias temáticas nas quais estão expressos os principais conceitos que os ACSs possuem sobre introdução de alimentos complementares, sendo que uma dessas categorias permitiu novo reagrupamento das suas unidades de análise temática, isto é, a construção de subcategorias. Essas informações, contidas nos fragmentos das falas dos participantes, foram confrontadas com o que diz a literatura sobre o tema contemplado pelo estudo.

Para assegurar o anonimato dos participantes utilizaram-se códigos compostos pelas iniciais da expressão Agente Comunitário de Saúde (A.C.S.) seguido por números ímpares, em sequência, que indicam a ordem em que os sujeitos foram entrevistados, que são: A.C.S.1, A.C.S.3, A.C.S.5, A.C.S.15.

Após identificação dos conceitos inadequados e das dúvidas mais relevantes pertinentes ao tema, elaborouse uma estratégia educativa, que consistiu em uma palestra ministrada aos ACSs, relacionada à introdução de uma alimentação complementar saudável no primeiro ano de vida, abrangendo aspectos como período de introdução, composição, consistência e modo de preparo da alimentação ser ofertada. $\mathrm{Na}$ ocasião, foi entregue a cada um dos participantes a versão impressa dos "Dez passos para uma alimentação saudável" ${ }^{7}$, para crianças menores de dois anos.

Finalmente, aplicou-se um questionário com estes participantes, objetivando avaliar a eficiência da estratégia de ação implementada e que mudanças ela imprimiu na percepção dos mesmos sobre o tema. A aplicação desse questionário ocorreu ao término da capacitação, pois devido à grande dificuldade em conseguir reunir os participantes para a estratégia educativa, o tempo para conclusão da análise de dados, conforme cronograma pré-estabelecido, ficou restrito. Este fato constituiu-se em uma limitação do estudo, uma vez que inviabilizou a avaliação da ação educativa em longo prazo.

O projeto de pesquisa foi submetido ao Comitê de Ética em Pesquisa (CEP) da Universidade Regional do Cariri, sendo aprovado sob parecer de no 48/2010. O Termo de Consentimento Livre e Esclarecido foi assinado por todos os participantes após terem sido informados acerca dos objetivos do estudo.

\section{RESULTADOS E DISCUSSÃO}

\section{Caracterização dos sujeitos}

Os sujeitos do estudo foram caracterizados de forma que no grupo constituído pelos oito agentes comunitários de saúde, identificaram-se sete do sexo feminino e um participante do sexo masculino, apresentando idade entre 21 e 45 anos, com média de 35,7. Seis dos entrevistados possuíam ensino médio completo e dois, ensino superior completo. Com relação ao tempo de trabalho desenvolvido na UBS, houve uma média de aproximadamente quatro anos de serviço.

\section{Categorização temática das falas}

Posteriormente, mediante leitura exaustiva dos dados, buscando-se uma maior compreensão das falas, verificaram-se elementos comuns nos discursos dos participantes, os quais foram dispostos em duas categorias temáticas: alimentação complementar após os seis meses de vida na ótica dos agentes comunitários de saúde e conseqüências de uma introdução alimentar inadequada, totalizando 131 unidades de análise temáticas, sendo que a primeira categoria encontra-se distribuída em três subcategorias: composição e idade de introdução da alimentação complementar; noções concernentes à consistência e modo de preparo da alimentação fornecida e conceitos sobre os alimentos que não devem ser oferecidos.

Neste sentido, vale ressaltar que as categorias 
supracitadas, as quais serão descritas a seguir, referemse à análise e interpretação das informações contidas nas entrevistas, antes da ação educativa realizadas com os sujeitos.

Categoria I: Alimentação complementar após os seis meses de vida na ótica dos agentes comunitários de saúde

Nesta categoria, composta pelas três subcategorias descritas abaixo, os sujeitos discorrem sobre as características de uma alimentação complementar saudável, ressaltando aspectos que vão desde composição, preparação e período de introdução da alimentação complementar até que alimentos não devem ser oferecidos.

Composição e idade de introdução da alimentação complementar

Nesta subcategoria, os ACSs citam os grupos alimentares que devem estar presentes na alimentação complementar, conceituando-a como a oferta de outros alimentos à criança após os seis meses de vida, como mostram as falas:

“Alimentação complementar... É aquela que completa. Não é a principal, é a que completa... Vai completar a principal. [...] غ̇ depois dos seis meses... (A.C.S.13).

Eu acredito que é a alimentação depois que passa o período, só amamentando né? Até os seis meses. Então aí vai começando a introduzir os novos alimentos na criança (A.C.S.7).

Infere-se pelos discursos que há um correto entendimento sobre 0 que significa alimentação complementar assim como seu período de introdução e seu caráter complementar em relação ao aleitamento materno. Esta compreensão é fundamental, uma vez que a introdução precoce ou tardia de outros alimentos e a interrupção do aleitamento materno nesta ocasião pode expor a criança ao risco nutricional ${ }^{8,9}$. Posteriormente, os agentes comunitários de saúde passaram a apontar quais alimentos deveriam compor a dieta infantil nesse período.
É... Nós procuramos com aquela alimentação mais balanceada como, no geral, verduras, frutas. [...] alimentos mais simples, não tão pesados (A.C.S.11).

$\mathrm{Na}$ sopinha, os legumes. Geralmente tem que se colocar cenoura, batatinha... Um franguinho né? De início... Verdura... (A.C.S.15).

A partir do sexto mês de vida deve-se introduzir uma dieta adequada, pautada numa alimentação composta por quantidades equilibradas de macronutrientes e micronutrientes encontrados nos principais grupos de alimentos como carnes, ovo, frutas, verduras, legumes, leguminosas, hortaliças, tubérculos e cereais ${ }^{10}$. As refeições salgadas devem, pois, ser variadas compreendendo um alimento de cada um desses grupos citados ${ }^{11}$.

No entanto, notou-se pelos discursos que há um conhecimento reduzido acerca dos principais grupos de alimentos que devem integrar a alimentação complementar, sendo comentados apenas os grupos dos legumes, verduras e frutas. Além disso, ao fazer referências ao grupo das carnes, os participantes demonstraram certa restrição com relação à escolha da carne a ser ofertada, apontando o frango como principal opção, em detrimento de outros tipos como boi, peixe, miúdos e vísceras.

Noções concernentes à consistência e modo de preparo da alimentação fornecida

Nesta subcategoria, os depoentes expressam ideias concernentes à consistência que a alimentação complementar deve ter, bem como modo de preparo indicado. Durante leitura minuciosa das falas, pôde-se perceber que a maioria dos entrevistados definiu a apresentação pastosa dos alimentos como a mais indicada.

É o seguinte: é assim... as papinhas de frutas amassadinhas, nunca liquidificadas. Amassada porque aí vai ajudar ele a desenvolver a mastigação, [...] porque aí ele vai assimilando o gosto (A.C.S.9).

Geralmente o povo gosta de passar no liquidificador, mas eu acho melhor é amassar no garfo, na colher... Porque dizem que perde os nutrientes. Não sei 
se isso é verdade, mas é o que eu já ouvi falar (A.C.S.5).

A introdução de alimentação complementar espessa, em forma de papas e purês (alimentação pastosa), possui maior densidade energética do que sucos e sopas, o que garante à criança um correto aporte calórico num período em que a mesma ingere pouca quantidade de alimento devido pequena capacidade gástrica, além de estimular as funções de lateralização da língua e de mastigação ${ }^{12}$.

Em contrapartida, alguns participantes declararam orientar as mães ou cuidadores a passar os alimentos na peneira, acreditando que essa conduta estimulará e facilitará o processo de mastigação. Já para outros, o liquidificador seria a melhor opção, na medida em que facilitaria a deglutição.

[...] é... A sopinha deve ser peneirada... Assim, eu não oriento a passar no liquidificador, eu oriento às mães a peneirar, pra não perder nenhuma vitamina, ta entendendo? (A.C.S.1).

Tem que passar por um processo pra... No caso, salada de fruta, é bom passar por essa etapa aí do liquidificador, pra ficar melhor pra eles ingerirem (A.C.S.11).

Estes dados assemelham-se aos encontrados em um estudo qualitativo realizado com mães de crianças menores de um ano e profissionais de saúde, a partir do qual observou-se que tanto os profissionais quanto as mães utilizaram, frequentemente, em seus discursos o tema liquidificadores e peneiras para processamento dos alimentos, estando essa prática relacionada à redução da densidade energética da refeição, podendo causar déficit do ganho de peso ${ }^{13}$.

Neste sentido, constatou-se que os discursos divergem do que afirma a literatura sobre o assunto, uma vez que os modos de preparo mencionados alteram significativamente a consistência da dieta, dificultando a assimilação das diferentes composições alimentares pela criança e contribuindo para um maior índice de rejeição.

Finalmente, houve dois participantes que enalteceram a questão da oferta de sucos á criança a partir do sexto mês, alegando que esta não está apta a receber alimentos mais sólidos.

O suco... Você vai usar muito o suco né? Pra introdução... Até porque a criança ta acabando de sair do peitim e ela vai ter mais a facilidade de ir se adaptando com o suco até chegar a comer a comidinha mais sólida (A.C.S.15).

Eu acho que a partir dos seis... Depois dos seis meses, você já deve introduzir alguns alimentos, principalmente sucos [...] (A.C.S.3).

Sabe-se, porém que o consumo de sucos, mesmo os naturais devem ser limitado, pois possuem menor teor energético que a própria fruta ${ }^{14}$. Assim, os sucos, principalmente aqueles ricos me vitamina $C$ devem ser utilizados em pequenas doses, de preferência após as refeições principais, para aumentar absorção do ferro inorgânico presente nos vegetais folhosos ${ }^{15}$, não devendo substituí-las conforme os discursos supracitados.

Em suma, a partir dos discursos citados, deduziu-se que não há um consenso entre os sujeitos pesquisados quanto ao preparo dos alimentos, embora a maior parte dos participantes aponte os alimentos pastosos como àqueles mais indicados na ocasião da introdução alimentar

Conceitos sobre os alimentos que não devem ser oferecidos

Nesta subcategoria, os entrevistados indicam aqueles alimentos que não devem compor a dieta do lactante na ocasião da introdução de novos alimentos. Dentre os alimentos apontados como nocivos à saúde nutricional da criança estão os doces, os sucos artificiais, os alimentos gordurosos e os industrializados.

Acho que muito doce, gordura demais, em excesso. [...] O doce devido ao diabetes né? [...] e a gordura no geral, porque vai prejudicar o coração a respiração... Você sabe (A.C.S.15).

Eu encontrei uma mãezinha uma vez, a criança tava com diarréia e ela tinha ido pro mercantil comprar 
aqueles suquinhos de garrafinha pra dar à criança, aí eu acho que isso não é legal... Com vários tipos de substâncias artificiais pra dá a uma criança pequenininha. [...] esse tipo de coisa industrializada não é bom (A.C.S.5).

O estabelecimento de hábitos alimentares se dá, principalmente, na fase em que a criança começa a receber as primeiras refeições ${ }^{16}$. Nesse período, o lactente passa a conhecer e apreciar aqueles alimentos que Ihes são oferecidos com maior frequência, ao passo que desenvolvem maior tendência de rejeitar os demais ${ }^{14}$. Comumente, estes costumes alimentares se perpetuam até a fase adulta ${ }^{17,18}$.

Neste sentido, a introdução de alimentos ricos em gordura, açúcar, corantes ou conservantes pode causar o desinteresse da criança em consumir alimentos saudáveis como frutas e verduras ${ }^{7}$.

Contudo, não obstante haver recomendações do Ministério da Saúde acerca dos alimentos que não devem ser ofertados à criança, nota-se que essa prática é bastante comum no período da lactância.

Segundo um estudo realizado com o intuito de avaliar as práticas e o consumo alimentar de lactentes de três metrópoles do Brasil, evidenciou-se que estas crianças recebiam alimentos contra indicados como doces industrializados, biscoitos recheados, macarrão instantâneo, pratos congelados semiprontos, refrigerantes e sucos artificiais de forma precoce ${ }^{19}$.

Assim, verificou-se que todos os ACSs entrevistados possuíam correto conhecimento sobre alguns dos alimentos que não devem ser oferecidos à criança nesse estágio do desenvolvimento, sendo que alguns deles estabeleceram, ainda, uma relação direta entre a utilização desses alimentos e os danos nutricionais associados.

\section{Categoria II: Consequências de uma introdução alimentar inadequada}

Nesta categoria, os participantes do estudo citam os principais danos à saúde da criança, advindos de uma introdução alimentar mal conduzida no período da lactância:
Porque se a mãe não souber introduzir a alimentação no bebê, ele pode ficar desnutrido [...] (A.C.S.1).

[...] se aquela alimentação não for rica em cálcio, vitamina, lógico que a criança vai ter dificuldade no crescimento, a dentição [...]. Da mesma forma que se for exagerada, ela vai ter problemas também, como obesidade, que é uma das mais que agente encontra, e outras mais (A.C.S.15).

Pode ser uma anemia... A criança não desenvolver em termo de peso né? Altura. (A.C.S.11).

Em estudo desenvolvido por Silva e Rodrigues ${ }^{20}$, no qual se fez uma revisão sistemática da literatura sobre fatores de risco para a obesidade infantil, evidenciou-se que as crianças em idade escolar que consomem alimentos de alta densidade energética apresentaram risco para sobrepeso ou obesidade ${ }^{20}$. Observou-se, ainda, que há um maior risco de obesidade para aqueles lactentes que tiveram um padrão de amamentação ineficaz, contrários aos pressupostos da OMS, e uma introdução precoce de açucares e outros alimentos.

Percebe-se, pois, que a ocorrência de obesidade no período da lactância é condicionada não somente pela quantidade de alimentos dados à criança (excessos alimentares), segundo sugerido pelos discursos, mas, principalmente, por questões como tipo de alimentação oferecida e período em que essa introdução ocorre.

Assim como a obesidade, a desnutrição está associada ao desmame e a introdução de alimentos complementares inadequados de maneira precoce, tornando necessário que família e comunidade repensem seus paradigmas acerca de aleitamento materno e oferta de novos alimentos ${ }^{21,22}$.

Novamente os discursos revelam um conhecimento superficial, por parte dos participantes do estudo, acerca dos diversos aspectos relacionadas à alimentação complementar oferecida, envolvidos no desenvolvimento dos problemas nutricionais citados, uma vez que grande parte dessas variáveis não foi mencionada. Neste sentido, em somente um dos discursos supracitados (A.C.S.15) fez-se correta associação entre a deficiência de micronutrientes (cálcio e vitaminas) e o consequente déficit de crescimento, bem como problemas de dentição. 
Sabe-se que a carência de micronutrientes no primeiro ano de vida pode estar vinculada a uma introdução precoce ou tardia de alimentos complementares. No primeiro caso, embora haja um ganho ponderal devido aumento da oferta de energia e proteínas da dieta, não há um fornecimento variado e completo de todos os micronutrientes necessário ao correto crescimento linear ${ }^{10}$.

Em contrapartida, a postergação da introdução de novos alimentos após os seis meses está igualmente vinculada à carência nutricional e, portanto, ao déficit de crescimento, pois neste caso, há também, uma diminuição na disponibilidade de micronutrientes, uma vez que os mesmos passam a ser oferecidos de maneira insuficiente pelo leite materno neste momento $^{16}$.

Para Vitolo ${ }^{12}$, "Desde a infância a ingestão de cálcio é importante, pois os ossos e os dentes estão se formando". Ainda de acordo com esta autora, as recomendações de cálcio para o primeiro ano de vida seguem os valores referentes à quantidade desse micronutriente no leite materno, tendo em vista que esse corresponde ao principal alimento no período de lactância ${ }^{12}$.

Quanto às vitaminas, estas desempenham importante função no desenvolvimento da criança. A vitamina $A$, encontrada no leite materno e em alimentos de origem animal (fígado, leite, queijo) e vegetal (cenoura, couve, pimentão, mamão), desempenha importante papel na visão, na pele, no sistema imunológico e no crescimento e desenvolvimento celular. Portanto, sua deficiência no primeiro ano de vida pode predispor o lactente a infecções, falhas no crescimento e xeroftalmia ${ }^{23}$.

A anemia foi outro importante problema nutricional citado nas falas. Após os seis meses, na ocasião da introdução de novos alimentos e consequente diminuição do padrão de amamentação, há uma depleção nas reservas de ferro e, portanto, maior risco de anemia ferropriva, tornando-se necessário buscar o correto aporte desse nutriente nos alimentos complementares ingeridos ${ }^{24,25}$.

Diante do exposto, conclui-se que apesar da maioria dos sujeitos entrevistados terem demonstrado conhecimento sobre os principais problemas nutricionais inerentes a uma incorreta introdução alimentar, notou-se na maioria destes discursos certa dificuldade em correlacionar essas complicações aos seus respectivos condicionantes, os quais estão associados à introdução alimentar insatisfatória e que podem ser: excesso ou insuficiência de alimentos oferecidos, alimentação inadequada em tipo e/ou consistência, dentre outros ${ }^{26}$.

\section{Impactos da ação educativa realizada}

Através da aplicação do questionário, após a realização da ação educativa, percebeu-se que houve melhora do nível de conhecimento sobre alimentação de transição por parte dos ACSs, uma vez que os mesmos conseguiram caracterizar, corretamente, uma alimentação complementar saudável, nos parâmetros variedade, consistência e modo de preparo dos alimentos.

Assim, todos os sujeitos souberam indicar, corretamente, os principais grupos de alimentos (leguminosas, legumes, frutas, verduras, carnes, dentre outros) que devem compor a alimentação oferecida e aqueles que não devem integrá-la como, refrigerantes, enlatados, chocolates, alimentos gordurosos, doces. Quanto à consistência e modo de preparo da alimentação, os sujeitos elegeram a consistência pastosa e os alimentos amassados com o garfo como conduta correta.

Por outro lado, somente $50 \%$ dos participantes souberam discorrer, satisfatoriamente, acerca dos principais problemas nutricionais advindos de uma alimentação complementar inapropriada, sendo que o restante citou apenas um ou dois dos vários distúrbios comumente encontrados. Estes dados indicam que apesar das mudanças de perspectiva e do aprimoramento de alguns conceitos, persistiu relativa dificuldade por parte de alguns dos participantes em enumerar os vários problemas nutricionais decorrentes de uma introdução alimentar inadequada, bem como seus múltiplos fatores condicionantes.

\section{CONSIDERAÇÕES FINAIS}

Percebeu-se que é deficiente o conhecimento dos agentes comunitários de saúde em relação à alimentação de transição infantil, apontando para a 
necessidade de aperfeiçoamento frente à temática por parte desses profissionais de saúde, capaz de propiciar melhora na qualidade das informações e, por conseguinte, das orientações prestadas à comunidade. Além disso, o desfecho favorável alcançado com a realização da estratégia educativa proposta pelo estudo, só fortalece a importância de haver essa educação continuada.

\section{REFERÊNCIAS}

1. Borelli M, Palma D, Taddei JAAC, Lopez FA. Fatores de risco associados ao desmame precoce e ao período de desmame em lactentes matriculados em creches. Rev Paul Pediatr. 2009;27(3):272-81.

2. Corrêa EN, Corso ACT, Kazapi I, Moreira EAM. Alimentação complementar e características maternas de crianças menores de dois anos de idade em Florianópolis/SC. Rev Paul Pediatr. 2009;27(3):258-64.

3. Saldiva SRDM, Escuder MM, Mondini L, Levy RB, Venancio SI, Escuder MML. Práticas alimentares de crianças de 6 a 12 meses e fatores maternos associados. J Pediatr. 2007;83(5):53-8.

4. Brasil. Ministério da Saúde. Política Nacional de Atenção Básica. [online]. $4^{\mathrm{a}}$ ed. Brasília (DF): Ministério da Saúde; 2007. (Série Pactos pela Saúde 2006; v. 4). [capturado 2010 jul 2]. Disponível em: http://bvsms.saude.gov.br/bvs/ publicacoes/politica_nacional_atencao_basica_4ed.pdf

5. Silva JA, Dalmaso ASW. O Agente Comunitário de Saúde e suas atribuições: os desafios para os processos de formação de recursos humanos em saúde. Interface Comun Saúde Educ. [periódico online] 2002 [capturado 2010 jun 6]; 6(10):75-96. Disponível em: http://www.scielo.br/scielo.php?pid=S1414-328320020001 00007\&script=sci arttext.

6. Bardin L. Análise de conteúdo. Lisboa: Edições 70; 2000.

7. Brasil. Ministério da Saúde. Dez passos para uma alimentação saudável. [online].2 $2^{\mathrm{a}}$ ed. Brasília (DF): Ministério da Saúde; 2010. (Normas e Manuais Técnicos.) [capturado 2011 mai 22]. Disponível em: http://www.rgnutri.com.br/sap/saudepublica/Dez\%20Passo s\%20Para\%20Uma\%20Alimentacao\%20Saudavel.pdf.

8. Silva LMP, Venancio SI, Marchioni DML. Práticas de alimentação complementar no primeiro ano de vida e fatores associados. Rev Nutr. 2010;23(6):983-92.

9. França MCT, Giugliani ERJ, Oliveira LD, Weigert E M L, Santo LCE, Köhler CV, Bonilha ALL. Uso de mamadeira no primeiro mês de vida: determinantes e influência na técnica de amamentação. Rev Saúde Púb. [periódico online]. 2008 [capturado 2010 jun 25];42(4):607-14. Disponível em: http://www.scielo.br/scielo.php?pid=S0034$89102008000400005 \&$ script=sci_arttext.

10. Oliveira LPM, Assis AMO, Pinheiro SMC, Prado MS, Barreto ML. Alimentação Complementar nos primeiros dois anos de vida. Rev Nutr PUCCAMP [periódico online]. 2005 [capturado 2011 mai 2];18(4):459-69. Disponível em: http://www.scielo.br/scielo.php?pid=s1415-527320050004 00002\&script=sci_arttext.

11. Moro GMB, Sartori F. Alimentação complement ar de crianças em aleitamento materno. Lecturas Educación Física y Deportes (Buenos Aires). [periódico online]. 2010 [capturado mai 5];15:1-5. Disponível em: http://www.efdeportes.com/efd151/alimentacaocomplementar-de-criancas-em-aleitamento.htm.
12. Vitolo MR. Práticas Alimentares na Infância. In: Vitolo MR. Nutrição: da gestação ao envelhecimento. Rio de Janeiro: Rubio; 2008.

13. Ferreira JV, Castro LMC, Garcia MFM. Alimentação no primeiro ano de vida: a conduta dos profissionais e a prática exercida pela família. Rev Ceres [periódico online]. 2009 [capturado 2013 maio 7]; 4(3):117-29. Disponível em: http://www.nutricao.uerj.br/revista/v4n3/pdf/artigo2. pdf.

14. Sarni R O S. Alimentação no Primeiro Ano de Vida. Rev Pediatr Mod. 2007;43(3):121-9.

15. Brasil. Ministério da Saúde. Saúde da criança: nutrição infantil. Brasília (DF): Ministério da Saúde; 2009. Normas e Manuais Técnicos. [capturado 2010 jun 6]. Disponível em: http://bvsms.saude.gov.br/bvs/publicacoes/saude crianca_nutricao_aleitamento_alimentacao.pdf.

16. Ribeiro JF, Marinho PCM, Valezin DF, Ballestero E, Aparecido J C, Costa L F V. Instrumento educativo sobre alimentação de lactentes: baseado nas necessidades de conhecimento das mães. Rev Inst Ciênc Saúde [periódico online] 2009 [capturado 2010 maio 23];27(1):7-11. Disponível em: http://www.unip.br/comunicacao/ publicacoes/ics/edicoes/2009/01_jan-mar/V27_N1_p1117.pdf.

17. Arimatea JE, Castro LMC, Rotenberg S. Práticas alimentares de crianças menores de um ano: as orientações de profissionais de saúde e as recomendações do Ministério da Saúde. Rev Ceres. [periódico online]2009 [capturado 2011 fev 12];4(2):65-78. Disponível em: http://www.nutricao.uerj.br/revista/ v4n2/art_2.htm.

18. Bercini LO, Masukawa MLT, Martins MR, Labegalini MPC, Alves NB. Alimentação da criança no primeiro ano de vida em Maringá, PR. Ciênc Cuid Saúde. [periódico online]. 2007 [capturado 2011 fev 10]; 6(Supl 2):404-10 Disponível em: http://periodicos.uem.br/ojs/index.php/ CiencCuidSaude/article/view/5339/3386

19. Caetano MC, Ortiz TTO, Silva SGLS, Souza FIS, Sarni ROS. Alimentação complementar: práticas inadequadas em lactentes. Jorn Pediatr. 2010;86(3):196-201.

20. Silva D P, Rodrigues E. Obesidade/sobrepeso infantil e seus fatores de risco: uma revisão de literatura. Rev Min Educ Fís. (UFV).[online] 2010 [capturado 2011 mar 6];5:136-46. Disponível em: http://www.revistamineiradeefi.ufv.br/artigos/arquivos/2b20 20e91a8c7eb0bde3f36a42ef33c8.pdf.

21. Silveira FJ, Lamounier JA. Prevalência do aleitamento materno e práticas de alimentação complementar em crianças até 24 meses de idade na região do alto Jequitinhonha, Minas Gerais. Rev Nutr. (Campinas). [online] 2004 [capturado 2011 abr 30];17(4):437-47. Disponível em: http://www.scielo.br/scielo.php? script=sci_arttext\&pid=S1415-52732004000400004.

22. Frota MA, Barroso MGT. Repercussão da desnutrição infantil na família. Rev Latinoam Enferm. (Ribeirão Preto).[periódico online] 2005 [capturado 2011 mai 3]; 13 (6):996-1000. Disponível em: http://www.scielo.br/ scielo.php?script=sci_arttext\&pid=S0104-1169200500060 0012.

23. Martins MC, Santos LMP, Lima AP, Santana LA, Araújo MPN, Santos SMC. Avaliação de políticas públicas de segurança alimentar e combate à fome no período 19952002: 3-Programa de Combate à Deficiência de Vitamina A. Cad Saúde Pública [periódico online] 2007 [capturado 2011 mai 8];23(9):2081-93. Disponível em: http://www.scielo.br/scielo.php?script=sci_arttext\&pid=S01 02-311X2007000900016. 
24. Bortolini GA. Anemia Ferropriva. In: Vitolo MA. Nutrição: da gestação ao envelhecimento. Rio de Janeiro (RJ): Rubio; 2008. p.243-56.

25. Almeida PC, Castro LMC, Damião JJ. Alimentação complementar oportuna e consumo de alimentos ricos em ferro e facilitadores de sua absorção no município do Rio de Janeiro. Rev Ceres. [periódico online]. 2010 [capturado 2011 fev 20];5(1):5-17. Disponível em: http://www.nutricao.uerj.br/revista/v5n1/pdf/art_1.pdf.

26. Silva $R Q$, Gubert $M B$. Qualidade das informações sobre aleitamento materno e alimentação complementar em sites brasileiros de profissionais de saúde disponíveis na internet. Rev Bras Saúde Matern Infant. 2010;10(3):33140.

\section{Endereço para correspondência:}

Pábula Parente Correia

Rua Onze de Fevereiro $n^{\circ} 384$

Recife/PE - CEP 50640-340

Telefone: +558184156806

E-mail: ppc_jesus@hotmail.com 